\title{
Survey of joint mobility and in vivo skin elasticity in London schoolchildren
}

\author{
S. SILVERMAN, L. CONSTINE, W. HARVEY, AND R. GRAHAME \\ From Guy's Arthritis Research Unit, Guy's Hospital, London, SE1 9RT
}

\begin{abstract}
Silverman, S., Constine, L., Harvey, W., and Grahame, R. (1975). Annals of the Rheumatic Diseases, 34, 177. Survey of joint mobility and in vivo skin elasticity in London schoolchildren. A survey of joint mobility was conducted in 295 healthy children between the ages of 5 and 10 years who attended a London primary school. Estimates of the commonly used measurements, that is passive dorsiflexion of the wrists and ankles, passive hyperextension of the elbows and knee, were too insensitive to detect any age effect. However, a method of estimating extensibility of the 5th metacarpophalangeal joint in response to a standard load detected a highly significant inverse correlation between joint mobility and age in the samples tested $(r=-0.586 ; \mathrm{P}<0.0001)$. There was no apparent sex difference.

Skinfold thickness using the Harpenden caliper over the right 3rd metacarpal bone and the in vivo skin elasticity measured using a suction cup device performed on a sample of 78 of the children revealed no influence of either age or sex on these parameters. This is in sharp contradistinction to the effect of both age and sex in these two parameters in adults. Furthermore, no correlation could be established between joint flexibility and skin elasticity in this study. Ligamentous laxity and skin hyperextensibility concur in certain hereditary disorders of connective tissue, e.g. the Ehlers-Danlos syndrome. However, in this study of healthy children, no simple relationship was apparent between the restraining effect of joint ligaments on the one hand and resistance to skin stretch (i.e. elasticity) on the other, despite the fact that the tensile properties of both these tissues devolve almost exclusively on collagen. The possible reasons for this seemingly anomalous result are discussed.
\end{abstract}

Although joint hypermobility in its extreme form may be associated with a hereditary disorder of connective tissue, it is now widely acknowledged that normal subjects exhibit varying degrees of joint laxity and that so-called hypermobile subjects merely represent the upper end of a normal spectrum of joint mobility. Clinical interest has been aroused by recent observations that a number of complications may supervene in hypermobile joints (Table I), resulting from increased ligamentous laxity and

\section{Table I Consequences of hypermobility}

(1) Predisposition to trauma (Nicholas, 1970)

(2) Recurrent dislocation (Macleod, 1882)

(3) Recurrent effusions (Sutro, 1947)

(4) Baker's cyst formation (Grahame, 1971)

(5) Backache (Howes and Isdale, 1971)

(6) Musculoskeletal symptoms (Kirk, Ansell, and Bywaters, 1967; Beighton, Solomon, and Soskolne, 1973) irrespective of cause. Progress in the understanding of the clinical significance of hypermobility and a knowledge of its epidemiology has been marred by the lack of precise criteria for measuring joint mobility. Carter and Wilkinson (1964) devised a simple scoring system based on the ability of the subject to perform certain passive manoeuvres. These were later modified by Beighton and Horan (1969).

These methods were crude and had certain serious shortcomings. These included their inability to distinguish between different grades of hypermobility and the fact that in the case of some joints minor degrees of hypermobility could be overlooked altogether. This failing was illustrated in the case of the 5th metacarpophalangeal joint in a previous study (Grahame and Jenkins, 1972). One intrinsic difficulty in measuring ranges of passive movement is that the observed range depends on the force applied to the moving part which is likely to vary both with the enthusiasm of the observer and the pain threshold of the subject. 
This is illustrated by the following experiment which we undertook on ourselves. With the hand clamped to the bench the angle of passive dorsiflexion of the 5th metacarpophalangeal joint was measured photographically in response to increasing loads applied to the volar surface of the corresponding distal interphalangeal joint. The result in three normal subjects is shown in Fig. 1. It will readily be appreciated that as the applied force is increased there is increasing dorsiflexion. That is to say, there is no cut-off point. This is so despite the fact that the experiment was continued in each case to the maximum load tolerated.

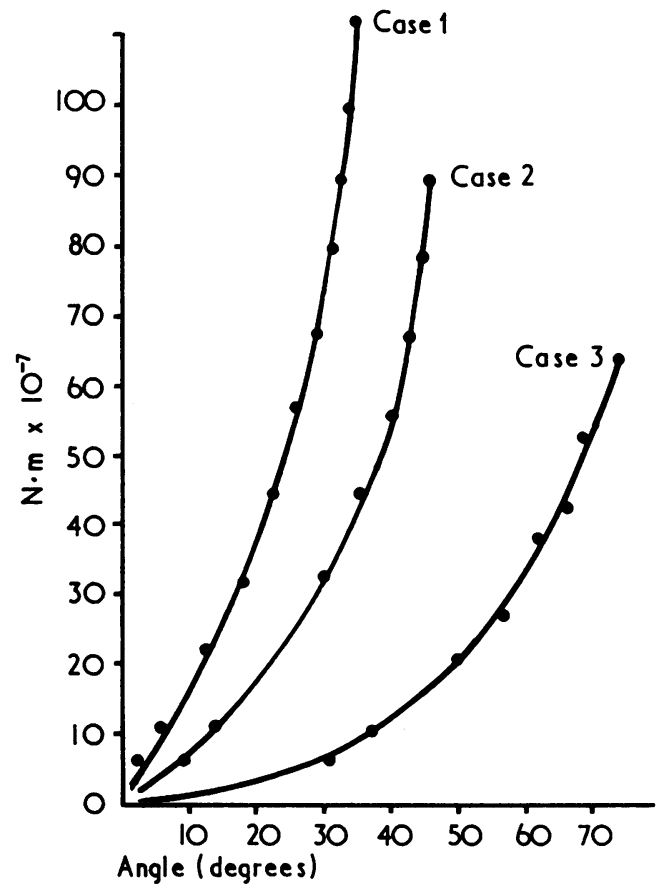

FIG. 1 Result of an experiment in three normal subjects to determine the extensibility of the 5th right metacarpophalangeal joint in response to increasing loads. Case 3 was clinically hypermobile

It follows from the result of this experiment that some kind of standardization of the applied load is a prerequisite of any method designed to measure joint mobility with precision.

\section{Method}

The present study was undertaken to explore the wellknown decline in joint mobility that takes place during childhood and to determine which, if any, of a variety of methods of measurement are the most sensitive. Subsequently, the study was extended to investigate the relationship between joint mobility and in vivo skin elasticity, since it is widely held that these parameters are related to the integrity of the collagen in joint ligament and dermis, respectively.

The original study of joint mobility was carried out on 295 healthy children of both sexes who attended a South London primary school. All tests were undertaken with the full consent of the children, their parents, and the school authorities concerned.

The following quantitative measurements were made.

(1) Passive dorsiflexion of the wrist.

(2) Passive hyperextension of the elbow.

(3) Passive hyperextension of the knee.

(4) Passive dorsiflexion of the ankle. (In each case the mean of two independent estimations was made on each pair of joints with a goniometer, or in the case of the ankle a modified protractor.)

(5) An estimate of passive dorsiflexion of the 5th metacarpophalangeal joint was made in response to a predetermined load of $1 \mathrm{lb}(4.44 \mathrm{~N})$ applied to the volar aspect of the distal interphalangeal skin crease by a pushpull tension gauge and measured with a protractor. This technique has been described in an earlier communication (Grahame and Jenkins, 1972) and a study of its intra- and interobserver error is published as an Appendix to this paper. In each case the mean of two independent observations was taken and the distance between the creases overlying the distal interphalangeal and the metacarpophalangeal joints was measured with a ruler. In this way it was possible to express extensibility of the joint in degrees $/ 10^{-7} \mathrm{~N} \cdot \mathrm{m}$.

(6) Since the range of joint movement may also be limited by tension in muscle, provision was made in this study for the simple assessment of hamstring muscle tension as follows. With the subject lying supine and the hip of the limb being tested flexed to $90^{\circ}$, the knee was slowly extended until resistance was encountered. At this point the angle between the thigh and the calf was measured with a goniometer.

In the second part of the study skin thickness and skin elasticity estimations were made on a sample of 79 children selected at random from each age and sex group of the main study. Skin thickness was estimated using the Harpenden caliper on a skinfold overlying the 4th right metacarpal shaft (Tanner and Whitehouse, 1955). The skin elasticity was measured by the in vivo suction cup method (Grahame and Holt, 1969; Grahame, 1970).

\section{Results}

The results are shown in Table II. There was no observable age influence in respect of passive dorsiflexion of the wrist, ankle, hyperextension of the elbow or knee. No change in hamstring tension was observed. Hyperextensibility of the metacarpophalangeal joint showed a highly significant inverse correlation with age (Fig. 2); but no difference between boys and girls or between dominant and nondominant hand was apparent with this parameter.

Despite the fact that our previous studies had shown a significant age and sex effect on skin thickness and in vivo elasticity in adults no such effects were apparent in the sample of children tested (Table II). 
Table II A. Results of joint mobility estimations in 295 children according to age

\begin{tabular}{|c|c|c|c|c|c|c|c|}
\hline & $5 y r s$ & $6 y r s$ & $7 y r s$ & $8 y r s$ & $9 y r s$ & 10 yrs & $P$ \\
\hline $\begin{array}{l}\text { 5th MCP extensibility } \\
\left.\text { (degrees } / 10^{-5} \mathrm{~N} \bullet \mathrm{m}\right)\end{array}$ & $5 \cdot 9 \pm 0 \cdot 2$ & $5 \cdot 4 \pm 0 \cdot 2$ & $4 \cdot 9 \pm 0 \cdot 2$ & $4 \cdot 5 \pm 0 \cdot 3$ & $4 \cdot 0 \pm 0 \cdot 3$ & $3 \cdot 8 \pm 0 \cdot 4$ & $<0.0001$ \\
\hline $\begin{array}{l}\text { Wrist extension (deg) } \\
\text { Elbow hyperextension (deg) } \\
\text { Knee hypertension (deg) } \\
\text { Ankle dorsiflexion (deg) } \\
\text { Hamstring tension (deg) }\end{array}$ & $\begin{array}{c}74 \pm 7 \\
7 \pm 3 \\
7 \pm 4 \\
59 \pm 7 \\
165 \pm 10\end{array}$ & $\begin{array}{c}75 \pm 7 \\
10 \pm 5 \\
6 \pm 3 \\
59 \pm 7 \\
161 \pm 10\end{array}$ & $\begin{array}{r}73 \pm 8 \\
7 \pm 4 \\
7 \pm 4 \\
60 \pm 6 \\
160 \pm 9\end{array}$ & $\begin{array}{r}75 \pm 9 \\
7 \pm 4 \\
6 \pm 4 \\
62 \pm 6 \\
163 \pm 13\end{array}$ & $\begin{aligned} & 75 \pm 10 \\
& 5 \pm 5 \\
& 3 \pm 3 \\
& 66 \pm 6 \\
& 160 \pm 11\end{aligned}$ & $\begin{array}{c}80 \pm 11 \\
7 \pm 5 \\
3 \pm 2 \\
65 \pm 8 \\
160 \pm 11\end{array}$ & $\begin{array}{l}\text { NS } \\
\text { NS } \\
\text { NS } \\
\text { NS } \\
\text { NS }\end{array}$ \\
\hline
\end{tabular}

B. Results of skin elasticity and skin thickness estimations in 78 children according to age and sex

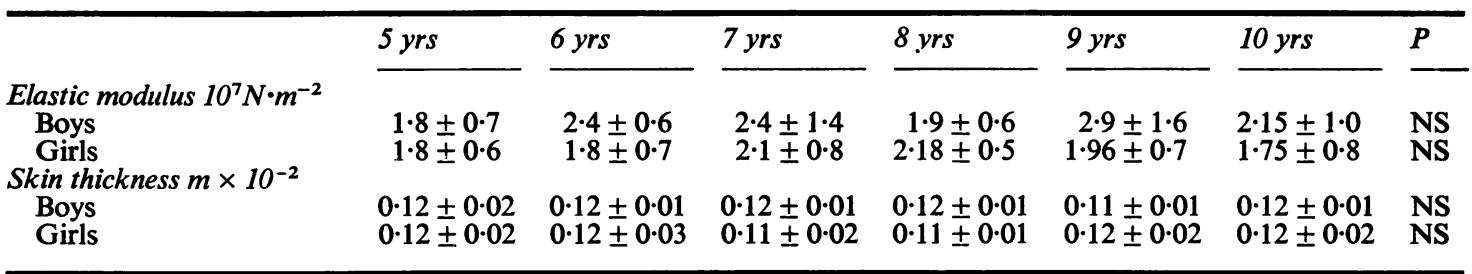

Furthermore, there was no correlation between extensibility of the 5 th metacarpophalangeal joint and the elastic modulus for skin (boys $r=0 \cdot 147$; $P>0 \cdot 1 \mathrm{NS}$; girls $r=0 \cdot 207 ; \mathrm{P}>0 \cdot 1 \mathrm{NS})$.

\section{Discussion}

An explanation for the absence of correlation between joint mobility and skin elasticity may be found in an earlier study of skin elasticity performed on a patient suffering from the Ehlers-Danlos syndrome, compared with a healthy control (Grahame and Beighton, 1969, Fig. 3), when it was shown that the elastic modulus as represented by the gradient of

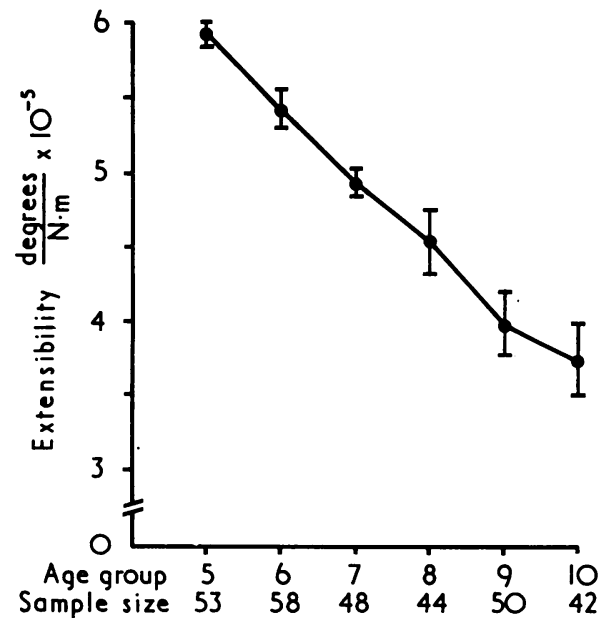

FIG. 2 Extensibility of the 5th metacarpophalangeal joint related to age, showing a highly significant inverse correlation $(r=-0.586 ; P<0.0001)$ the upper part of the stress-strain curve was almost identical with that of the control. The physical abnormality in the Ehlers-Danlos syndrome appeared to arise in the early phase of extension, seen in the lower part of the curve and corresponding with the taking up of slack. Comparing this graph (Fig. 3) with that of the joint experiment described above (Fig. 1) reveals a strikingly similarity. Moreover, it will be appreciated that the cut-off point

\section{- Ehlers-Danlos syndrome \\ - Control subject}

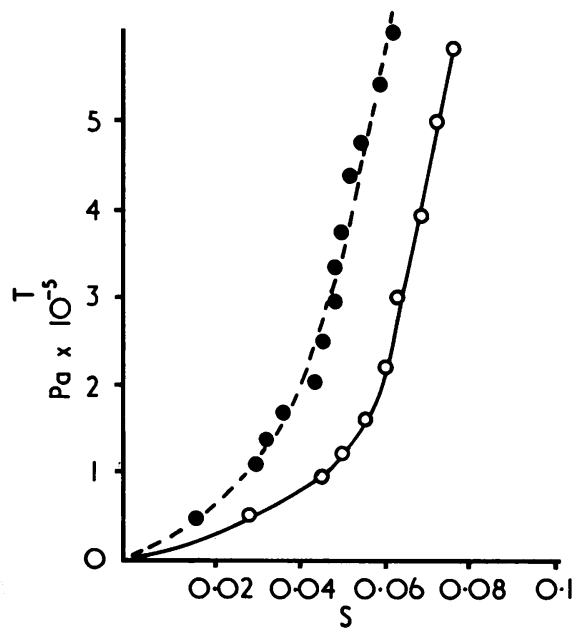

FIG. 3 Stress/strain curve for intact skin from a patient suffering from the Ehlers-Danlos syndrome. (Reproduced from Fig. 5 of Grahame and Beighton (1969) with kind permission of the Editor of Annals of the Rheumatic Diseases) 
taken when measuring the extensibility of the metacarpophalangeal joint falls within the range of 'taking up of slack' of the joint capsular ligaments. One is simply precluded from exploring the range representing the elasticity of joint collagen by the advent of pain. It is not, therefore, surprising that observations of extensibility of this joint fail to correlate with skin elasticity data obtained from the same subjects.

The results suggest that extensibility of the 5th metacarpophalangeal joint as measured by the method described is the most sensitive index of joint ligamentous laxity available. Its sensitivity has been confirmed in the present study by the demonstration of a progressive decline in joint mobility in childhood between the ages of 5 and 10 years, although this decline was not apparent when the conventional methods were used.
We wish to thank the Inner London Education Authority for permitting us to undertake this study and all the children who took part for their willing collaboration. W.H. was in receipt of a grant from Armour Pharmaceutical Co.

\section{Appendix}

The individual observer error involved in the measurement of flexibility of these joints was determined on six healthy volunteer subjects. Ten consecutive measurements were performed on each relevant joint in each subject, and the mean error expressed as the coefficient of variation (see Table III). This is the ratio of standard deviation to the mean and is expressed as a percentage.

The measurement of flexibility was highly reproducible in the 5th metacarpophalangeal joint and the wrist, and progressively decreased in the ankle, knee, hamstring, and elbow, respectively.

Table III Mean coefficient of variation in 6 volunteers; 10 consecutive observations by each observer

\begin{tabular}{|c|c|c|c|c|c|c|}
\hline Observer & 5th $M C P$ & Wrist & Elbow & Knee & Ankle & Hamstring \\
\hline $\begin{array}{l}1 \\
2 \\
3\end{array}$ & $\begin{array}{l}4 \cdot 5 \\
5 \cdot 6 \\
5 \cdot 3\end{array}$ & $\begin{array}{l}2 \cdot 4 \\
4 \cdot 1 \\
5 \cdot 4\end{array}$ & $\begin{array}{l}47 \\
46 \\
53\end{array}$ & $\begin{array}{l}30 \\
29 \\
33\end{array}$ & $\begin{array}{l}20 \\
8 \cdot 3 \\
20\end{array}$ & $\begin{array}{l}32 \\
24 \\
12\end{array}$ \\
\hline
\end{tabular}

\section{References}

Beighton, P., ANd Horan, F. (1969) J. Bone Jt Surg., 51B, 444 (Orthopaedic aspects of the Ehlers-Danlos syndrome)

-, Solomon, L., AND Soskolne, C. L. (1973) Ann. rheum. Dis., 32, 413 (Articular mobility in an African population)

Carter, C., AND Wilkinson, J. (1964) J. Bone Jt Surg., 46B, 40 (Persistent joint laxity and congenital dislocation of the hip)

Grahame, R. (1970) Clin. Sci., 39, 223 (A method for measuring human skin elasticity in-vivo with observations on the effects of age, sex and pregnancy) (1971) Proc. roy. Soc. Med. ,64, 692 (Joint hypermobility-clinical aspects)

- and Beighton, P. (1969) Ann. rheum. Dis., 28, 246 (Physical properties of the skin in the Ehlers-Danlos syndrome)

- AND Holt, P. J. (1969) Gerontologia, 15, 121 (The influence of ageing on the in-vivo elasticity of human skin)

-, AND Jenkins, J. M. (1972) Ann. rheum. Dis., 31, 109 (Joint hypermobility-asset or liability? A study of joint mobility in ballet dancers)

Howes, R. G., AND ISDALE, I. C. (1971) Rheum. \& Phys. Med., 11, 72 (The loose back: an unrecognised syndrome)

Kirk, J. A., ANSell, B. M., AND Bywaters, E. G. L. (1967) Ann. rheum. Dis., 26, 419 (The hypermobility syndrome)

MACLEOD, G. H. B. (1882) Glasgow med. J., 17, 343 (The case of Charles H. Warren)

NicholaS, J. A. (1970) J. Amer. med. Ass., 212, 2236 (Injuries to knee ligaments)

Surro, C. J. (1947) Surgery, 21, 67 (Hypermobility of bones due to 'overlengthened' capsular and ligamentous tissues)

Tanner, J. M., ANd Whitehouse, R. H. (1955) Amer. J. Phys. Anthrop., 13, 743 (The Harpenden skin fold caliper) 